Research Paper

\title{
S1P promotes IL-6 expression in osteoblasts through the PI3K, MEK/ERK and NF- $\kappa$ B signaling pathways
}

\author{
Sung-Lin $\mathrm{Hu}^{1,2 \#, ~ C h i e n-C h u n g ~ H u a n g}{ }^{1,3 \#}$, Tzu-Ting Tzeng4, Shan-Chi Liu ${ }^{5}$, Chun-Hao Tsai ${ }^{6,7}$, Yi-Chin \\ Fong ${ }^{6,8}$ and Chih-Hsin Tang $1,4,9,10^{\bowtie}$ \\ 1. School of Medicine, China Medical University, Taichung, Taiwan \\ 2. Department of Family Medicine, China Medical University Hsinchu Hospital, Hsinchu, Taiwan \\ 3. Division of Immunology and Rheumatology, Department of Internal Medicine, China Medical University Hospital, Taichung, Taiwan \\ 4. Graduate Institute of Biomedical Sciences, China Medical University, Taichung, Taiwan \\ 5. Department of Medical Education and Research, China Medical University Beigang Hospital, Yunlin, Taiwan \\ 6. Department of Sports Medicine, College of Health Care, China Medical University, Taichung, Taiwan \\ 7. Department of Orthopedic Surgery, China Medical University Hospital, Taichung, Taiwan \\ 8. Department of Orthopedic Surgery, China Medical University Beigang Hospital, Yunlin, Taiwan \\ 9. Chinese Medicine Research Center, China Medical University, Taichung, Taiwan \\ 10. Department of Biotechnology, College of Health Science, Asia University, Taichung, Taiwan \\ \# These authors contributed equally. \\ $\triangle$ Corresponding authors: Chih-Hsin, Tang PhD, Department of Pharmacology, School of Medicine, China Medical University, Taichung, Taiwan; E-mail: \\ chtang@mail.cmu.edu.tw. Yi-Chin, Fong MD, PhD, Department of Orthopedic Surgery, China Medical University Hospital, Taichung, Taiwan; E-mail: \\ yichin.fong@gmail.com
}

(C) The author(s). This is an open access article distributed under the terms of the Creative Commons Attribution License (https://creativecommons.org/licenses/by/4.0/). See http://ivyspring.com/terms for full terms and conditions.

Received: 2020.02.06; Accepted: 2020.04.27; Published: 2020.05.18

\begin{abstract}
Rheumatoid arthritis (RA) is a systemic autoimmune inflammatory disease, in which the immune system attacks joint tissue. Interleukin (IL)-6 is a key proinflammatory cytokine in RA progression. Sphingosine-1-phosphate (SIP), a platelet-derived lysophospholipid mediator, reportedly regulates osteoimmunology. Here, we examined the effects of SIP on IL-6 expression in osteoblasts. Our results and records from the Gene Expression Omnibus (GEO) database demonstrate higher levels of IL-6 in patients with RA compared with those with osteoarthritis. Stimulation of osteoblasts with SIP increased $m R N A$ and protein expression of IL-6. PI3K, MEK, ERK and NF- $\kappa$ B inhibitors and their small interfering RNAs (siRNAs) reduced SIP-promoted IL- 6 expression. SIP also facilitated PI3K, MEK/ERK and NF- $\kappa$ B signaling cascades. Our results indicate that SIP promotes the expression of IL-6 in osteoblasts via the $\mathrm{PI3K}, \mathrm{MEK} / \mathrm{ERK}$ and NF- $\kappa \mathrm{B}$ signaling pathways.
\end{abstract}

Key words: S1P; IL-6; Osteoblasts; Arthritis

\section{Introduction}

Rheumatoid arthritis (RA) is a systemic autoimmune inflammatory disease, in which the immune system attacks joint tissue [1, 2]. An inflammatory response, pannus development, synovial swelling, joint stiffness and articular cartilage degradation are major symptoms of RA [3]. Thus, much RA research has concentrated on synovial inflammation and cartilage destruction [4]. Few reports have focused on the effects of subchondral bone erosions in RA pathogenesis [5-7]. Accumulating evidence suggests that osteoblasts, the most abundant cell type in subchondral bone, play an important role in cartilage pathology during the development of RA $[8,9]$. Thus, elucidation of the molecular mechanism of osteoblasts is a critical issue for RA treatment.

Interleukin (IL)-6, a T-cell-derived cytokine that promotes the maturation of $B$ cells into antibody-secreting cells, involves multiple biological activities and plays a critical role in innate and acquired immune responses, cancer progression and metastasis, inflammatory reactions, as well as osteoclast generation [10], and is well recognized as an important proinflammatory cytokine during RA progression [11, 12]. The therapeutic success of the 
IL-6 inhibitor tocilizumab in RA has encouraged the development of other IL-6 inhibitors, only one of which (sarilumab) has so far received EU and US regulatory approval for the treatment of RA [13].

Sphingosine-1-phosphate (S1P), a plateletderived lysophospholipid mediator, reduces plateletderived growth factor (PDGF)-promoted chemotaxis and cellular Rac activation [14]. The S1P/S1P receptor axis regulates several biologic functions such as tumor invasion and progression, angiogenesis and vasculogenesis as well as skeletal muscle and nervous system degeneration [15-17]. Interestingly, deleting the $\mathrm{S}_{1} \mathrm{P}_{2}$ receptor promotes murine embryonic fibroblast migration towards S1P and also PDGF, which stimulates $\mathrm{S} 1 \mathrm{P}$ production; $\mathrm{S} \mathrm{P}_{2}$ deletion also increases the enzymatic expression and activity of sphingosine kinase 1 (SphK1), which is responsible for producing S1P [18]. In addition, S1P/S1P signaling reportedly regulates osteoimmunology [19] and upregulation of the S1P receptor in synovial tissues has found in the collagen-induced arthritis model [20]. However, the role of S1P in IL-6 expression and RA pathogenesis is uncertain. We describe how we found that S1P promotes IL-6 expression in human osteoblasts and also involvement of the PI3K, MEK/ERK and NF-KB signaling pathways in S1P-promoted IL-6 production.

\section{Materials and Methods}

\section{Materials}

We obtained antibodies against p-PI3K, p-MEK, p-ERK, p-p65, PI3K, MEK, ERK, p65 and $\beta$-actin from Santa Cruz Biotechnology (CA, USA). S1P was purchased from Avanti Polar Lipid Inc. (Alabaster, AL, USA). ON-TARGETplus siRNAs were purchased from Dharmacon Research (Lafayette, CO, USA). Gibco-BRL Life Technologies (Grand Island, NY, USA) supplied fetal bovine serum (FBS) and all other cell culture reagents. Promega (Madison, WI, USA) supplied the pSV- $\beta$-galactosidase vector and luciferase assay kits. The $\kappa \mathrm{B}$ luciferase plasmid was purchased from Stratagene (La Jolla, CA). The human IL-6 promoter constructs were provided by Dr. Oliver Eickelberg (Department of Medicine II, University of Giessen, Giessen, Germany). All other chemicals or inhibitors were purchased from Sigma-Aldrich (St. Louis, MO, USA).

\section{Human synovial fluids}

We obtained approval for this study from the local ethics committee (the Institutional Review Board of China Medical University Hospital) and all patients gave written informed consent before participating in this study. Abnormal synovial fluids were obtained from patients undergoing total knee arthroplasty for osteoarthritis (OA) or RA.

\section{Analysis of the Gene Expression Omnibus (GEO) database}

Gene expression profile records were obtained from the GEO database for analysis of IL-6 expression in OA and RA tissue samples [21].

\section{Cell culture}

Osteoblast-like cell lines MG-63 and MC3T3-E1 were purchased from American Type Culture Collection (Manassas, VA, USA). Cells were cultured in MEM supplemented with $10 \%$ FBS and antibiotics then maintained in a humidified incubator at $37^{\circ} \mathrm{C}$ in $5 \% \mathrm{CO}_{2}$.

\section{Western blot analysis}

Extracted proteins were resolved by SDS-PAGE and transferred to Immobilon ${ }^{\circledR}$ PVDF membranes. Western blot analysis was performed according to our previous reports [22-24].

\section{Quantitative real-time PCR (qPCR)}

Total RNA was extracted from osteoblasts using TRIzol reagent. qPCR analysis was conducted according to an established protocol [25-27].

\section{Enzyme-Linked Immunosorbent (ELISA) assay}

Osteoblasts were treated with S1P alone for $24 \mathrm{~h}$ or pretreated with pharmacological inhibitors, followed by $24 \mathrm{~h}$ of S1P stimulation. The conditioned medium (CM) was collected and quantified for IL-6 levels using an IL-6 ELISA kit (Peprotech, Rocky Hill, NJ, USA), as per the manufacturer's protocol.

\section{Luciferase reporter assay}

Osteoblasts were co-transfected with $0.8 \mu \mathrm{g}$ IL-6 or $\kappa B$-luciferase reporter gene construct and $0.4 \mu \mathrm{g}$ $\beta$-galactosidase using Lipofectamine 2000, as per the manufacturer's instructions. After $24 \mathrm{~h}$ of transfection, the cells were exposed to S1P. Luciferase activity was determined using the luciferase assay kit [28-30].

\section{Statistics}

All values are presented as the mean \pm standard deviation (SD). Differences between the two experimental groups were assessed for significance using the Student's $t$-test. The difference was considered to be significant if the $p$ value was $<0.05$.

\section{Results}

\section{Upregulation of IL-6 expression in RA synovial fluid}

The proinflammatory role of IL-6 is well understood during RA pathogenesis [13]. To further confirm the role of IL-6 in RA angiogenesis, we first 
analyzed IL-6 expression in RA patients. We found that levels of IL-6 were markedly higher in synovial fluids from patients with RA compared with those from OA patients (Fig. 1A). Analyses of tissue samples from the GEO database also revealed higher levels of IL-6 mRNA expression amongst RA patients than in OA patients (Fig. 1B), suggesting that IL-6 plays a more important role in RA than in OA.

\section{S1P facilitates IL-6 expression}

S1P modulates osteoimmunology by targeting both osteoclastogenesis and osteogenesis [19]. We sought to determine whether S1P also regulates IL-6 expression in osteoblasts (MG-63 cells). Stimulation of osteoblasts with S1P concentration-dependently promoted IL-6 mRNA expression and protein secretion (Fig. 2A\&B). To confirm the role of S1P in IL-6 production, the cells were transfected with SphK1 siRNA, which diminished IL-6 expression (Fig. 2C). Similarly, the data from another osteoblast cell line (MC3T3-E1) showed that S1P increased IL-6 expression (Fig. 2D). S1P therefore appears to promote IL-6 production in osteoblasts.

\section{S1 P promotes IL-6 expression through the PI3K and MEK/ERK pathways}

The PI3K signaling pathway controls IL-6 production [31]. Treatment of cells with a PI3K inhibitor (Ly294402) reduced S1P-promoted IL-6 (Fig. 3A\&B). Transfection of the cells with PI3K siRNA reversed S1P-mediated effects (Fig. 3A\&B), while incubation of osteoblasts with S1P facilitated PI3K phosphorylation (Fig. 3C), indicating that PI3K is involved in S1P-enhanced IL-6 production.

MEK/ERK is a common signaling pathway for enhancing IL-6 expression [32]. Incubating osteoblasts

(A)

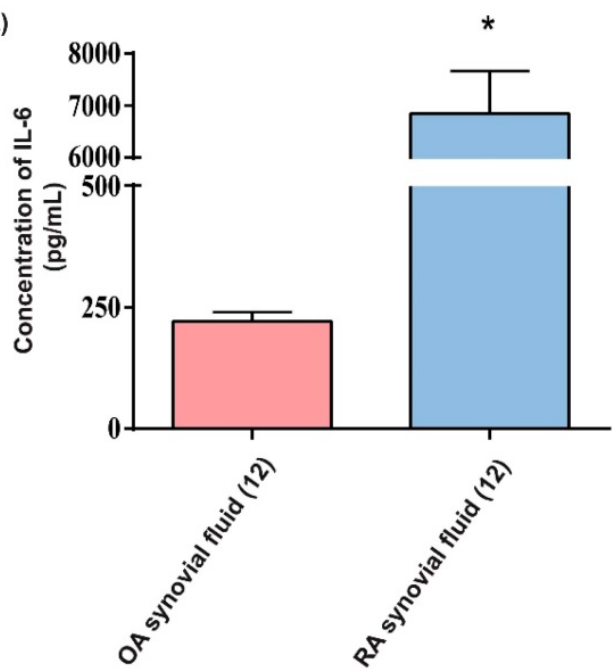

with a MEK inhibitor (U0126) and ERK inhibitor or siRNAs against MEK and ERK effectively inhibited S1P-promoted IL-6 expression (Fig. 4A\&B). Stimulation of osteoblasts by S1P increased MEK and ERK phosphorylation (Fig. 4C). These results suggest that S1P acts via PI3K and MEK/ERK signaling to enhance levels of IL-6 expression.

\section{NF- $\mathrm{B}$ transcriptional activity controls S1P-induced IL-6 production}

NF- $\kappa B$ appears to control IL-6 gene regulation [33]. We therefore examined whether NF-кB influences S1P-promoted IL-6 expression in osteoblasts. Treating cells with the NF- $\mathrm{KB}$ inhibitor PDTC or transfecting them with NF- $\mathrm{B}$ siRNA inhibited S1P-induced IL-6 production (Fig. 5A\&B). S1P significantly promoted NF- $\mathrm{BB}$ phosphorylation (Fig. 5C), which was reduced by pretreatment with PI3K, MEK and ERK inhibitors (Fig. 5D).

We further examined the effect of the NF- $\kappa B$ transcriptional binding site in the induction of IL-6 expression, using the IL-6 promoter construct pIL6-luc651 $(-651 /+1)$ and the NF-KB site mutation (pIL6-luc651 $\triangle \mathrm{NF}-\mathrm{kB}$ ). We found that S1P significantly increased pIL6-luc651 promoter activity, which was abolished by the NF- $\mathrm{BB}$ binding site mutation (Fig. 6A). To confirm that the PI3K and MEK1/ERK signaling pathway mediated S1P-enhanced activation

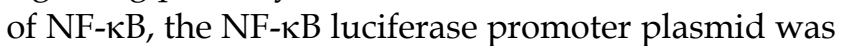
used. Treatment of cells with S1P augmented NF-кB luciferase activity, while pretreatment of the cells with PI3K, MEK and ERK inhibitors reduced S1P-induced NF- $\kappa$ B luciferase activity (Fig. 6B). Activation of PI3K MEK and ERK appears to be necessary for S1P-induced NF- $\kappa B$ activation in human osteoblasts.

(B)

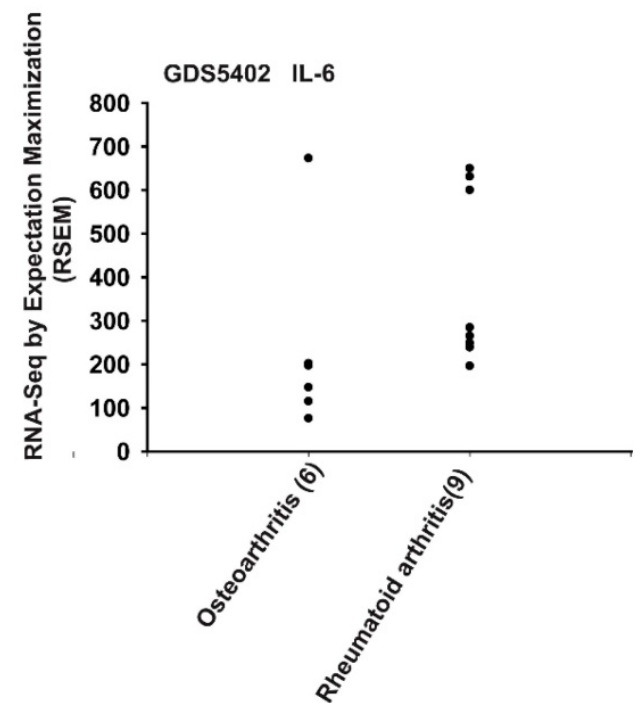

Figure 1: IL-6 levels are upregulated in RA patients. (A) IL-6 levels in synovial fluid from patients with OA and RA were quantified using the ELISA assay. (B) Levels of IL-6 expression in human RA and OA tissue samples obtained from the GEO database. Data represent the mean \pm S.D. 
(A)
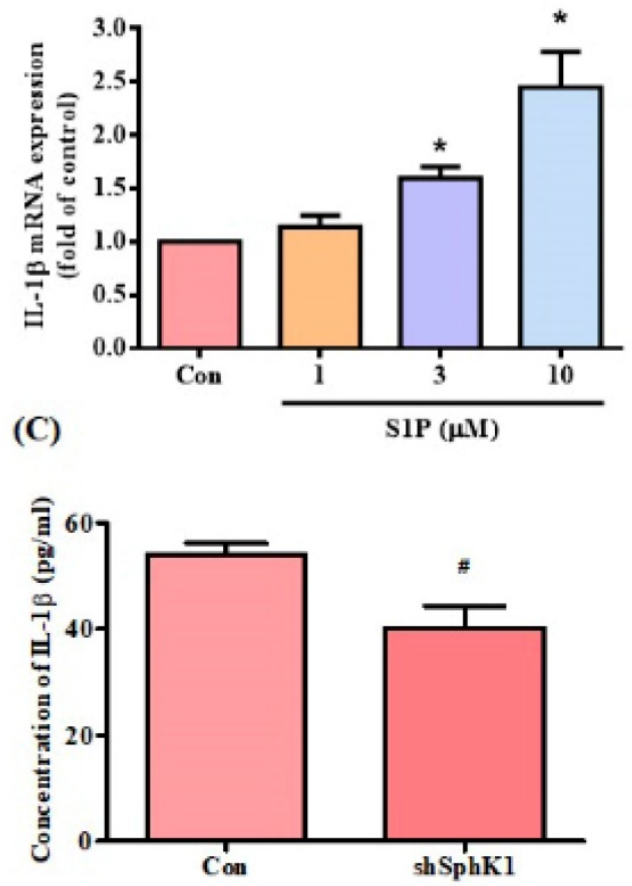

(B)
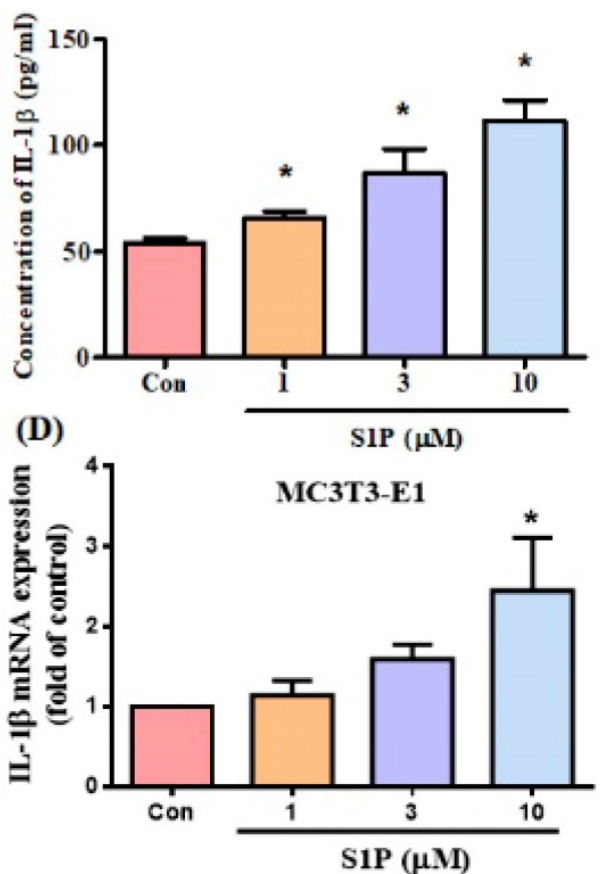

Figure 2: SIP promotes IL-6 expression in osteoblasts. MG-63 (A\&B) and MC3T3-E1 (D) cells were incubated with S1P (1-10 $\mu$ M) for 24 h; IL-6 expression was examined using the qPCR and ELISA assays. (C) MG-63 cells were transfected with SphK1 siRNA for 24 h; IL-6 expression was examined using the ELISA assay. Data represent the mean \pm S.D. $* p<0.05$ compared with the control group; \# $p<0.05$ compared with the control siRNA group.

(A)

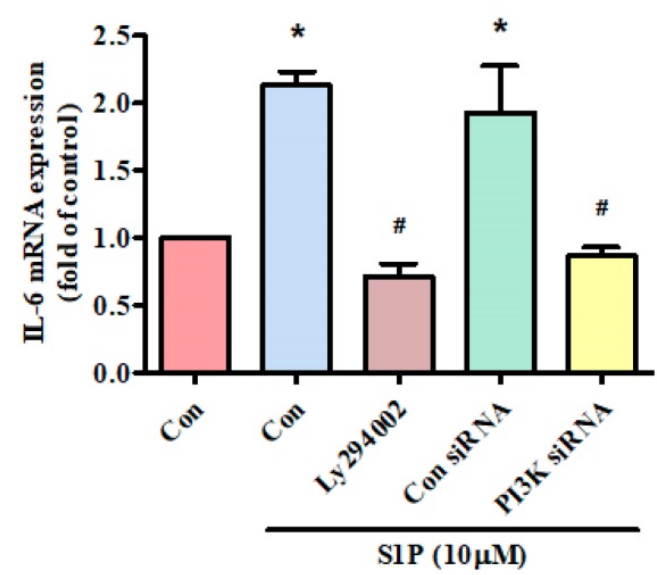

(B)

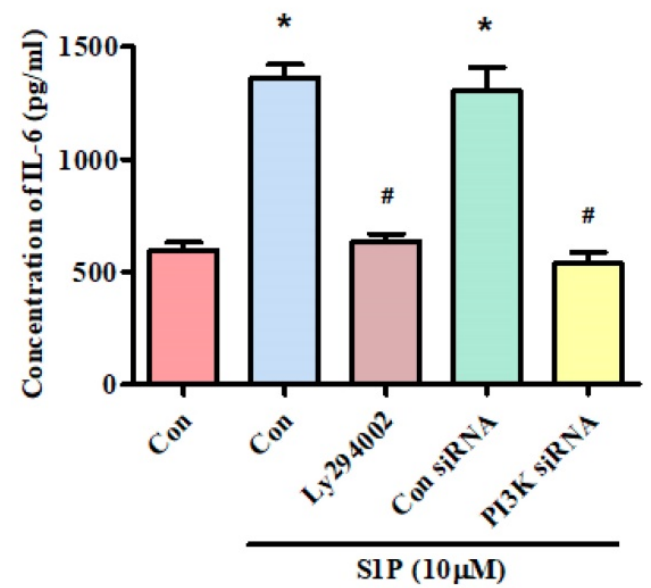

(C)

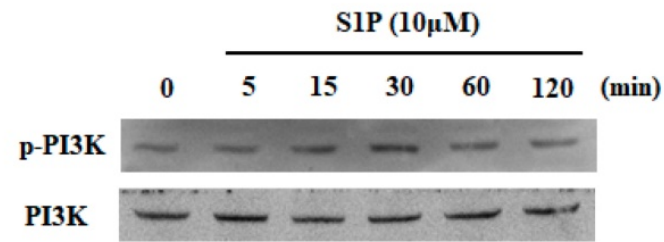

Figure 3: The PI3K pathway mediates SIP-promoted IL-6 expression. (A\&B) MG-63 cells were pretreated for 30 min with LY294002 or transfected with PI3K siRNA then stimulated with SIP. IL-6 expression was examined using the qPCR and ELISA assays. (C) MG-63 cells were incubated with S1P; PI3K phosphorylation was examined using the Western blot assay. Data represent the mean \pm S.D. $* p<0.05$ compared with the control group; \# $p<0.05$ compared with the SIP-treated group. 
(A)

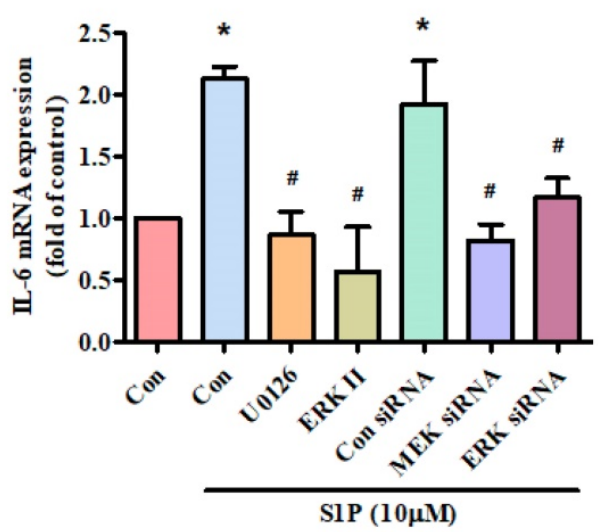

(C)

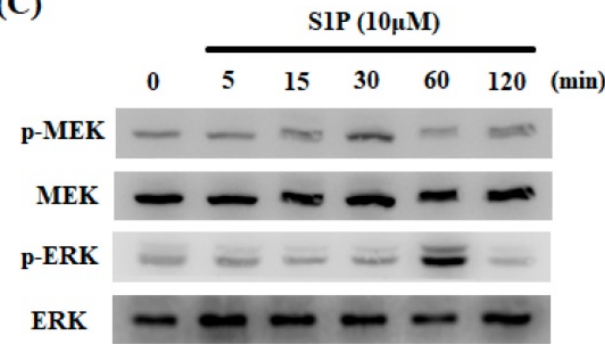

(B)

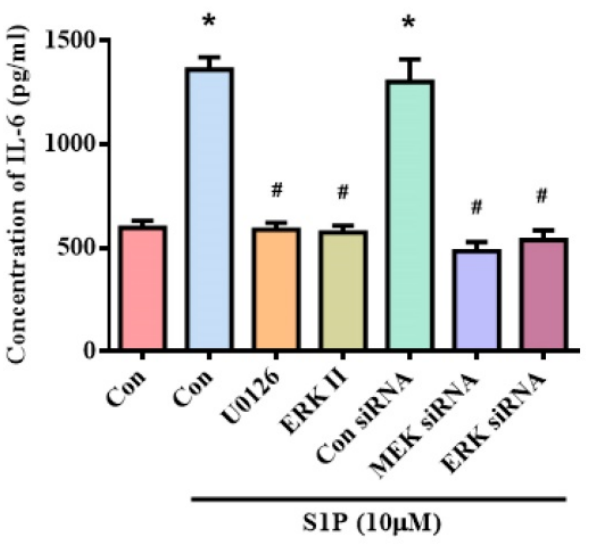

Figure 4: The MEK/ERK pathway is involved in SIP-induced IL-6 expression. (A\&B) MG-63 cells were pretreated for 30 min with U0126 and ERK inhibitor or transfected with MEK and ERK siRNAs then stimulated with SIP. IL-6 expression was examined by qPCR and ELISA assays. (C) MG-63 cells were incubated with SIP; MEK and ERK phosphorylation was examined using the Western blot assay. Data represent the mean \pm S.D. $* p<0.05$ compared with the control group; \# $p<0.05$ compared with the S1P-treated group.

(A)

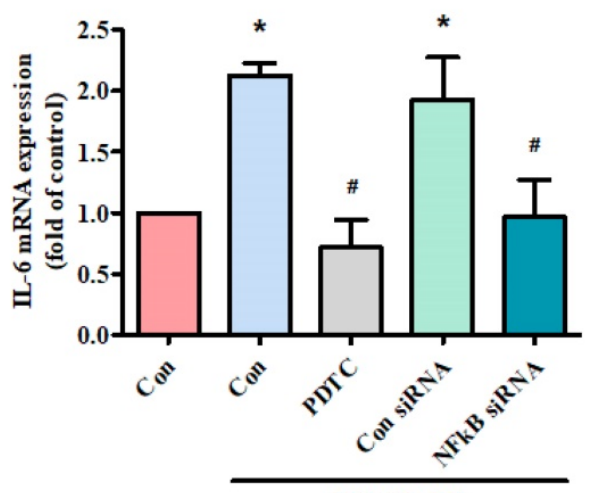

(C)

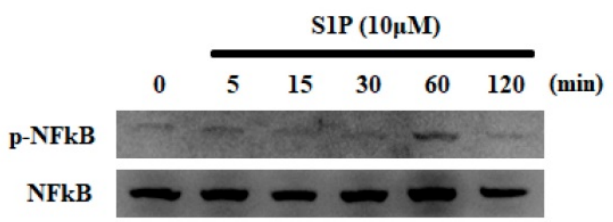

(B)

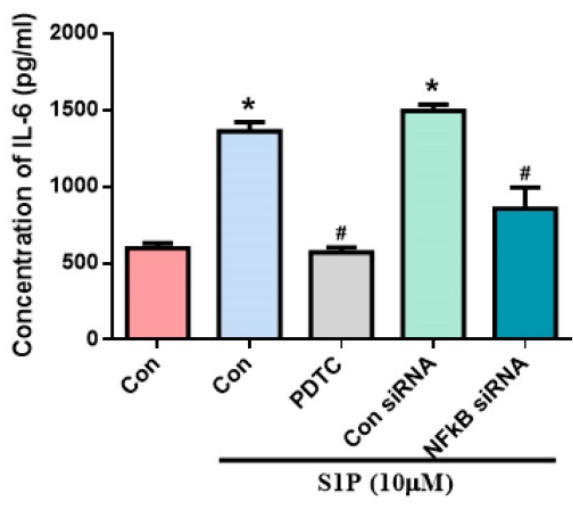

(D)

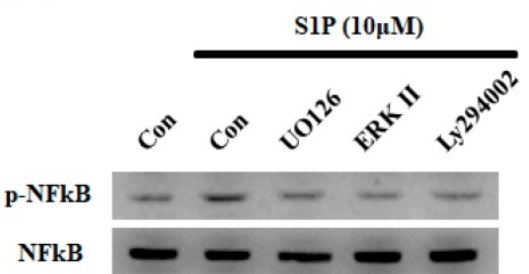

Figure 5: S1P increases IL-6 production through NF-KB activation. (A\&B) MG-63 cells were pretreated for 30 min with PDTC or transfected with NF- $K B$ siRNA then stimulated with S1P. IL-6 expression was examined by QPCR and ELISA assays. (C) MG-63 cells were incubated with SIP; NF- $\kappa B$ phosphorylation was examined using the Western blot assay. (D) MG-63 cells were pretreated with LY294002, U0126 or an ERK inhibitor for 30 min, then stimulated with S1P and NF-KB phosphorylation. Data represent the mean \pm S.D. $* p<0.05$ compared with the control group; \# $p<0.05$ compared with the S1P-treated group. 
(A)

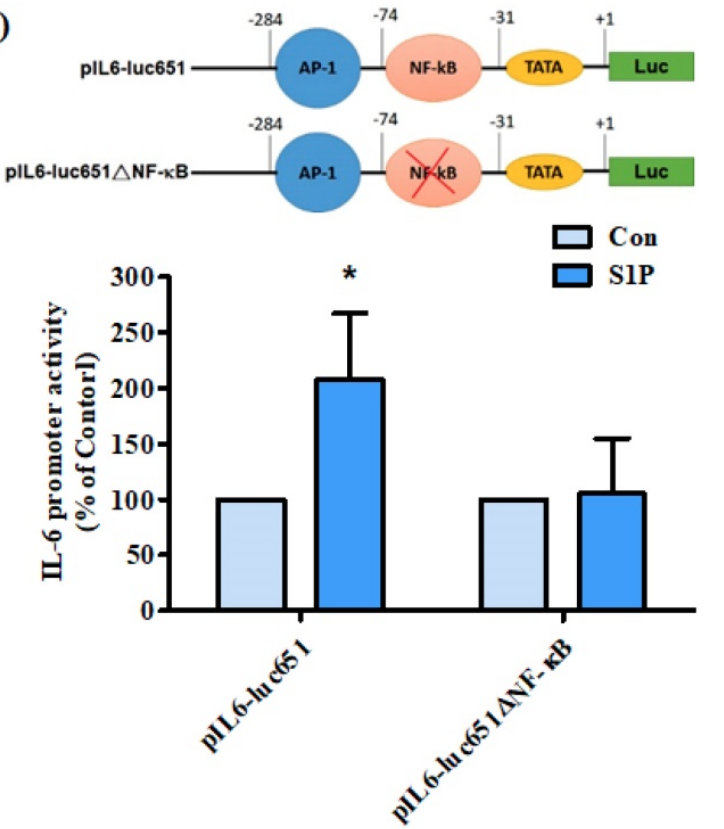

(B)

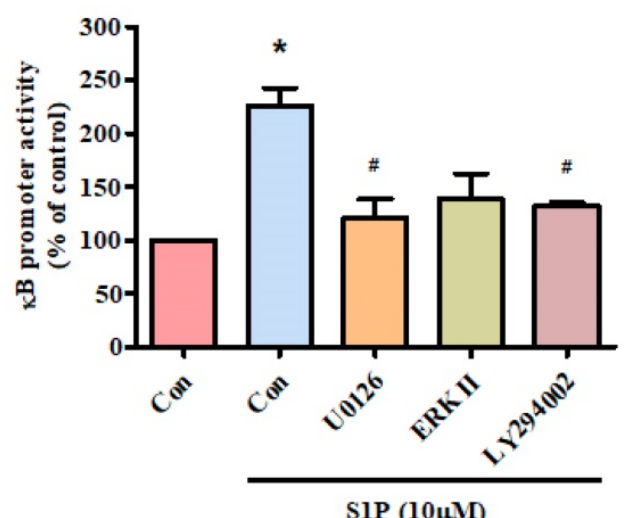

Figure 6: PI3K, MEK and ERK pathways are involved in SIP-facilitated NF-kB activity. (A) MG-63 cells were transfected with the indicated IL-6 luciferase plasmid and then stimulated with S1P. IL-6 luciferase activity was examined. (B) MG-63 cells were pretreated with LY294002, U0126 or an ERK inhibitor, then stimulated with S1P and NF- $\kappa B$ luciferase activity was examined. Data represent the mean \pm S.D. $* p<0.05$ compared with the control group; \# $p<0.05$ compared with the SIP-treated group.

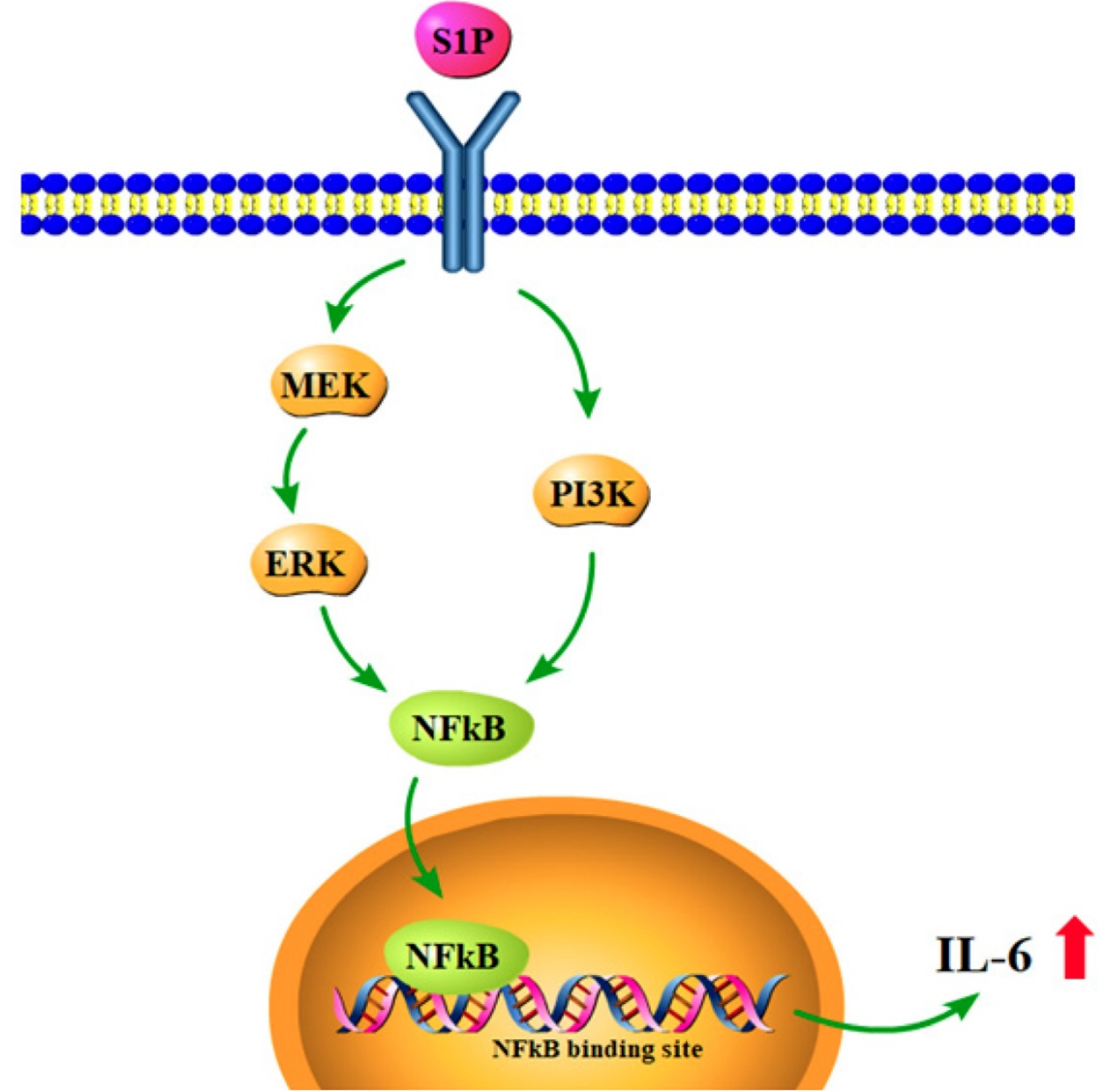

Figure 7. Schematic diagram summarizes the mechanisms of S1P-induced IL-6 production in osteoblasts. SIP promotes IL-6 expression in osteoblasts through the PI3K, MEK/ERK and NF-KB signaling pathways. 


\section{Discussion}

RA is associated with chronic synovial inflammation and cartilage degradation in the joints. The chronic inflammatory progression in RA is regulated through multiple cytokine networks. The factors responsible for initiating the degradation and loss of the articular tissues are largely unknown. Upregulation of S1P has been found in synovial tissues of the collagen-induced arthritis model [20]. However, little is known about the role of S1P in the expression of the critical inflammatory cytokine IL-6 and RA development. Here, we found that S1P increases IL-6 production in human osteoblasts through the PI3K, MEK/ERK and NF-кB signaling pathways.

IL-6 has numerous biological activities and previous research has revealed increased IL-6 concentrations in sera and synovial fluid from RA patients [34]. In this study, we confirmed that IL-6 levels are higher in synovial fluids from RA patients than from OA patients. Our analysis of records from the GEO database also demonstrated higher IL-6 levels in RA tissue than in OA tissue. We also identified IL-6 as a target protein for the SIP signaling pathway, which regulates inflammatory responses in RA disease. These results highlight the critical nature of IL-6 as a molecular target in RA therapy.

Accumulating evidence focuses on the important role of inflammation in osteoblasts, which exhibit critical effects within the arthritic bone microenvironment and thus in RA pathogenesis [35, 36]. The research suggests that the influence of inflammation in bone is specific to the site of inflammation and dependent on the cytokines present within the local bone microenvironment $[35,36]$. In this study, we found that S1P enhances IL-6 production in osteoblasts and that knockdown SphK1 diminishes IL-6 expression in osteoblasts. Our results provide evidence that inflammation in osteoblasts plays a critical role during the RA development.

The activation of the PI3K and MEK/ERK signaling pathway is essential for regulating multiple cellular functions [37]. These pathways also regulate IL-6 expression [33, 38]. In this study, we found that S1P facilitates PI3K, MEK and ERK phosphorylation, while PI3K, MEK and ERK inhibitors diminish S1P-enhanced IL-6 production. Furthermore, PI3K, MEK and ERK siRNAs confirmed the reversal of S1P-promoted IL-6 expression. It appears that the PI3K and MEK/ERK signaling pathways are involved in S1P-regulated IL-6 expression. Several binding sites exist for a number of transcription factors in the $5^{\prime}$ region of the $I L-6$ gene $[39,40]$. The results of this study show that a mutation of the NF-kB binding site inhibited S1P-enhanced IL-6 luciferase activity. In addition, an NF- $\mathrm{KB}$ inhibitor or siRNA reversed S1P-induced IL-6 expression. These results indicate that NF- $\kappa B$ activation is required for S1P-promoted IL-6 production. Our data also show that S1P facilitates NF- $\mathrm{BB}$ phosphorylation and luciferase activity. PI3K, MEK and ERK inhibitors all antagonized S1P-enhanced NF- $\mathrm{B}$ phosphorylation and luciferase activity, suggesting that the PI3K, MEK and ERK pathways regulate S1P-mediated NF- $\mathrm{BB}$ activation.

In conclusion, our study has identified that S1P promotes IL-6 production and inflammatory responses in osteoblasts via the PI3K, MEK/ERK and NF- $\kappa$ B signaling pathways (Fig.7). S1P appears to be a novel therapeutic target in RA.

\section{Acknowledgments}

This work was supported by grants from the Ministry of Science and Technology of Taiwan (MOST 108-2320-B-039-057; 108-2320-B-039-065-; 108-2314-B039-034 -MY3), China Medical University Hospital (DMR-109-236) and China Medical University Beigang Hospital (CMUBHR105-006). We would like to thank Iona J. MacDonald from China Medical University for her English language revision of this manuscript.

\section{Competing Interests}

The authors have declared that no competing interest exists.

\section{References}

1. Pap T, Korb-Pap A. Cartilage damage in osteoarthritis and rheumatoid arthritis-two unequal siblings. Nature reviews Rheumatology. 2015; 11: 606-15.

2. Choy EH, Panayi GS. Cytokine pathways and joint inflammation in rheumatoid arthritis. The New England journal of medicine. 2001; 344: 907-16.

3. MacDonald IJ, Liu SC, Su CM, Wang YH, Tsai CH, Tang CH. Implications of Angiogenesis Involvement in Arthritis. International journal of molecular sciences. 2018; 19.

4. Tang $\mathrm{CH}$. Research of Pathogenesis and Novel Therapeutics in Arthritis. International journal of molecular sciences. 2019; 20.

5. Murakami M, Nishimoto N. [IL-6 inhibitors prevent bone loss and cartilage degeneration in rheumatoid arthritis]. Clinical calcium. 2015; 25: 1851-7.

6. Dancevic CM, McCulloch DR. Current and emerging therapeutic strategies for preventing inflammation and aggrecanase-mediated cartilage destruction in arthritis. Arthritis research \& therapy. 2014; 16: 429.

7. Spreafico $R$, Rossetti $M$, van Loosdregt J, Wallace CA, Massa M, Magni-Manzoni S, et al. A circulating reservoir of pathogenic-like CD4+ T cells shares a genetic and phenotypic signature with the inflamed synovial micro-environment. Annals of the rheumatic diseases. 2014.

8. Chen CY, Su CM, Hsu CJ, Huang CC, Wang SW, Liu SC, et al. CCN1 Promotes VEGF Production in Osteoblasts and Induces Endothelial Progenitor Cell Angiogenesis by Inhibiting miR-126 Expression in Rheumatoid Arthritis. Journal of bone and mineral research : the official journal of the American Society for Bone and Mineral Research. 2017; 32: 34-45.

9. Su CM, Lee WL, Hsu CJ, Lu TT, Wang LH, Xu GH, et al. Adiponectin Induces Oncostatin M Expression in Osteoblasts through the PI3K/Akt Signaling Pathway. International journal of molecular sciences. 2015; 17.

10. Hunter CA, Jones SA. IL-6 as a keystone cytokine in health and disease. Nature immunology. 2015; 16: 448-57.

11. Tang $\mathrm{CH}$, Chen $\mathrm{CF}$, Chen WM, Fong YC. IL-6 increases MMP-13 expression and motility in human chondrosarcoma cells. The Journal of biological chemistry. 2011; 286: 11056-66. 
12. Wu MH, Tsai CH, Huang YL, Fong YC, Tang CH. Visfatin Promotes IL-6 and TNF-alpha Production in Human Synovial Fibroblasts by Repressing miR-199a-5p through ERK, p38 and JNK Signaling Pathways. International journal of molecular sciences. 2018; 19 .

13. Kang S, Tanaka T, Narazaki M, Kishimoto T. Targeting Interleukin-6 Signaling in Clinic. Immunity. 2019; 50: 1007-23.

14. Ryu Y, Takuwa N, Sugimoto N, Sakurada S, Usui S, Okamoto $\mathrm{H}$, et al. Sphingosine-1-phosphate, a platelet-derived lysophospholipid mediator, negatively regulates cellular Rac activity and cell migration in vascular smooth muscle cells. Circulation research. 2002; 90: 325-32.

15. Meacci E, Garcia-Gil M. S1P/S1P Receptor Signaling in Neuromuscolar Disorders. International journal of molecular sciences. 2019; 20.

16. Mascall KS, Small GR, Gibson G, Nixon GF. Sphingosine-1-phosphate-induced release of TIMP-2 from vascular smooth muscle cells inhibits angiogenesis. Journal of cell science. 2012; 125: 2267-75.

17. Tsai CH, Yang DY, Lin CY, Chen TM, Tang CH, Huang YL. Sphingosine-1-phosphate suppresses chondrosarcoma metastasis by upregulation of tissue inhibitor of metalloproteinase 3 through suppressing miR-101 expression. Molecular oncology. 2017; 11: 1380-98.

18. Goparaju SK, Jolly PS, Watterson KR, Bektas M, Alvarez S, Sarkar S, et al. The S1P2 receptor negatively regulates platelet-derived growth factor-induced motility and proliferation. Molecular and cellular biology. 2005; 25: 4237-49.

19. Xiao L, Zhou Y, Friis T, Beagley K, Xiao Y. S1P-S1PR1 Signaling: the "Sphinx" in Osteoimmunology. Frontiers in immunology. 2019; 10: 1409.

20. Inoue $\mathrm{T}$, Kohno $\mathrm{M}$, Nagahara $\mathrm{H}$, Murakami $\mathrm{K}$, Sagawa $\mathrm{T}$, Kasahara A, et al. Upregulation of sphingosine-1-phosphate receptor 3 on fibroblast-like synoviocytes is associated with the development of collagen-induced arthritis via increased interleukin-6 production. PloS one. 2019; 14: e0218090.

21. Lee HP, Wang SW, Wu YC, Lin LW, Tsai FJ, Yang JS, et al. Soya-cerebroside inhibits VEGF-facilitated angiogenesis in endothelial progenitor cells. Food Agr Immunol. 2020; 31: 193-204.

22. Lee HP, Chen PC, Wang SW, Fong YC, Tsai CH, Tsai FJ, et al. Plumbagin suppresses endothelial progenitor cell-related angiogenesis in vitro and in vivo. J Funct Foods. 2019; 52: 537-44.

23. Lee HP, Wang SW, Wu YC, Tsai CH, Tsai FJ, Chung JG, et al. Glucocerebroside reduces endothelial progenitor cell-induced angiogenesis. Food Agr Immunol. 2019; 30: 1033-45.

24. Liu JF, Lee CW, Tsai MH, Tang CH, Chen PC, Lin LW, et al. Thrombospondin 2 promotes tumor metastasis by inducing matrix metalloproteinase-13 production in lung cancer cells. Biochemical pharmacology. 2018; 155: 537-46.

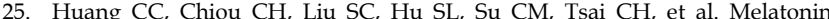
attenuates TNF-alpha and IL-1beta expression in synovial fibroblasts and diminishes cartilage degradation: Implications for the treatment of rheumatoid arthritis. Journal of pineal research. 2019; 66: e12560.

26. Liu SC, Tsai $\mathrm{CH}$, Wu TY, Tsai CH, Tsai FJ, Chung JG, et al. Soya-cerebroside reduces IL-1 beta-induced MMP-1 production in chondrocytes and inhibits cartilage degradation: implications for the treatment of osteoarthritis. Food Agr Immunol. 2019; 30: 620-32.

27. Wang M, Chao CC, Chen PC, Liu PI, Yang YC, Su CM, et al. Thrombospondin enhances RANKL-dependent osteoclastogenesis and facilitates lung cancer bone metastasis. Biochemical pharmacology. 2019; 166: 23-32.

28. Su CM, Tang CH, Chi MJ, Lin CY, Fong YC, Liu YC, et al. Resistin facilitates VEGF-C-associated lymphangiogenesis by inhibiting miR-186 in human chondrosarcoma cells. Biochemical pharmacology. 2018; 154: 234-42.

29. Yang YC, Chiou PC, Chen PC, Liu PY, Huang WC, Chao CC, et al. Melatonin reduces lung cancer stemness through inhibiting of PLC, ERK, p38, beta-catenin, and Twist pathways. Environmental toxicology. 2019; 34: 203-9.

30. Wu TJ, Lin $\mathrm{CY}$, Tsai $\mathrm{CH}$, Huang $\mathrm{YL}$, Tang $\mathrm{CH}$. Glucose suppresses IL-1beta-induced MMP-1 expression through the FAK, MEK, ERK, and AP-1 signaling pathways. Environmental toxicology. 2018; 33: 1061-8.

31. Chiu YC, Lin CY, Chen CP, Huang KC, Tong KM, Tzeng CY, et al. Peptidoglycan enhances IL-6 production in human synovial fibroblasts via TLR2 receptor, focal adhesion kinase, Akt, and AP-1- dependent pathway. Journal of immunology. 2009; 183: 2785-92.

32. Zegeye MM, Lindkvist M, Falker $\mathrm{K}$, Kumawat AK, Paramel G, Grenegard M, et al. Activation of the JAK/STAT3 and PI3K/AKT pathways are crucial for IL-6 trans-signaling-mediated pro-inflammatory response in human vascular endothelial cells. Cell communication and signaling: CCS. 2018; 16: 55.

33. Yang WH, Liu SC, Tsai CH, Fong YC, Wang SJ, Chang YS, et al. Leptin induces IL-6 expression through OBRl receptor signaling pathway in human synovial fibroblasts. PloS one. 2013; 8: e75551.

34. Lo SF, Huang CM, Lin HC, Chen WC, Tsai CH, Tsai FJ. Cytokine (IL-6) and chemokine (IL-8) gene polymorphisms among rheumatoid arthritis patients in Taiwan. Clinical and experimental rheumatology. 2008; 26: 632-7.

35. Huang $\mathrm{CY}$, Chen SY, Tsai $\mathrm{HC}$, Hsu HC, Tang $\mathrm{CH}$. Thrombin induces epidermal growth factor receptor transactivation and CCL2 expression in human osteoblasts. Arthritis and rheumatism. 2012; 64: 3344-54.

36. Su CM, Lee WL, Hsu CJ, Lu TT, Wang LH, Xu GH, et al. Adiponectin Induces Oncostatin M Expression in Osteoblasts through the PI3K/Akt Signaling Pathway. Int J Mol Sci. 2015; 17

37. Wee P, Wang Z. Epidermal Growth Factor Receptor Cell Proliferation Signaling Pathways. Cancers. 2017; 9.

38. Winkler C, Ferdous F, Dimmick M, Scott T. Lipopolysaccharide induced Interleukin-6 production is mediated through activation of ERK $1 / 2$, p38
MAPK, MEK, and NFkappaB in chicken thrombocytes. Developmental and comparative immunology. 2017; 73: 124-30.

39. Grassl C, Luckow B, Schlondorff D, Dendorfer U. Transcriptional regulation of the interleukin-6 gene in mesangial cells. Journal of the American Society of Nephrology : JASN. 1999; 10: 1466-77.

40. Lin CC, Chen KB, Tsai CH, Tsai FJ, Huang CY, Tang CH, et al. Casticin inhibits human prostate cancer DU 145 cell migration and invasion via Ras/Akt/NF-kappaB signaling pathways. Journal of food biochemistry. 2019; 43: e12902. 